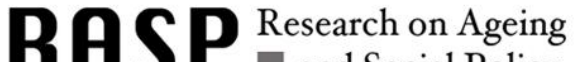
and Social Policy

Instructions for authors, subscriptions and further details:

http://rasp.hipatiapress.com

\title{
Análisis de la Dependencia en Población Geriátrica como Factor Pronóstico después de Sufrir Fractura de Cadera
}

Javier García Alba ${ }^{1}$, Ivan Dot Pascuet ${ }^{1}$, Alfredo Rodríguez Gangoso ${ }^{1}$, \& Gabriel De Febrer Martínez ${ }^{2}$

1) Grup Sagessa. Spain

2) Universitat Rovira i Virgili. Spain

Date of publication: July $30^{\text {th }}, 2019$

Edition period: July 2019- December 2019

To cite this article: García, J., Dot, I., Rodríguez, A. y De Febrer, G. (2019). Análisis de la Dependencia en Población Geriátrica como Factor Pronóstico después de Sufrir Fractura de Cadera. Research on Ageing and Social Policy, 7(2), 427-447. doi: 10.4471/rasp.2019.4445

To link this article: http://dx.doi.org/10.447/rasp.2019.4445

\section{PLEASE SCROLL DOWN FOR ARTICLE}

The terms and conditions of use are related to the Open Journal System and to Creative Commons Attribution License (CCAL). 


\section{Análisis de la Dependencia en Población Geriátrica como Factor Pronóstico después de Sufrir Fractura de Cadera}

Javier García Alba

Ivan Dot Pascuet

Alfredo Rodríguez Gangoso

Grup Sagessa
Gabriel De Febrer Martínez

Universitat Rovira i Virgili

(Recibido: 3 de julio 2019; Aceptado: 20 de julio 2019; Publicado: 30 de julio de 2019)

\section{Resumen}

En España se producen entre 50.000 y 60.000 fracturas de cadera al año. El objetivo de este análisis es evaluar la dependencia para las Actividades Básicas de la Vida diaria como factor pronóstico después de sufrir una fractura de cadera. Se realizó el análisis prospectivo de 428 pacientes ingresados por fractura de cadera entre los años 2015 y 2017. Se evaluó el grado de autonomía mediante el índice de Barthel previo al ingreso, la aparición de complicaciones durante el ingreso y la mortalidad a los 6 meses y al año. Un 43,6\% de los pacientes presentaban dependencia grave al ingreso según la escala de Barthel. La mortalidad a los 6 meses de seguimiento fue del $24 \%$ de los pacientes y al año del 33,5\%. El estudio de regresión logística muestra que sufrir dependencia grave en la escala de Barthel al ingreso es factor de riesgo de muerte a los 6 meses $(p=0,047)$ y el año $(p=0,043)$. Por otra parte, no sufrir dependencia es un factor protector de mortalidad a los 6 meses $(p=0,023)$.

Keywords: envejecimiento, dependencia, fractura de cadera, índice de Barthel

2019 Hipatia Press

ISSN: 2014-6728

DOI: $10.4471 /$ rasp.2019.4445
Hipatia Press

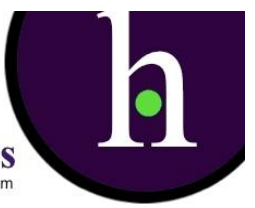




\section{Analysis of Dependency in Geriatric Population as a Prognostic Factor After Suffering Hip Fracture}

Javier García Alba

Ivan Dot Pascuet

Alfredo Rodríguez Gangoso

Grup Sagessa
Gabriel De Febrer Martínez

Universitat Rovira i Virgili

(Received: 3 July 2019; Accepted: 20 July 2019; Published: 30 July 2019)

\section{Abstract}

In Spain there are between 50,000 and 60,000 hip fractures a year. The objective of this analysis is to evaluate the dependency for the Basic Activities of Daily Life as a prognostic factor after suffering a hip fracture. We performed a prospective analysis of 428 patients admitted for hip fracture in our center between 2015 and 2017. The degree of autonomy was assessed using the Barthel index prior to admission, the appearance of complications during admission and mortality. at 6 months and a year. $43.6 \%$ of patients had severe dependence on admission according to the Barthel scale. The mortality at 6 months of follow-up was $24 \%$ of the patients and the year of $33.5 \%$. The logistic regression study shows that suffering severe dependence on the Barthel scale at admission is a risk factor for death at 6 months $(p=0.047)$ and the year $(p=0.043)$. On the other hand, not suffering from dependence is a protective factor of mortality at 6 months $(\mathrm{p}=0.023)$.

Palabras clave: aging, dependence, hip fracture, Barthel index

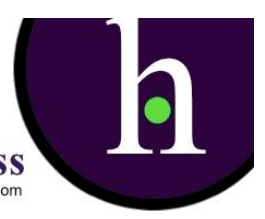




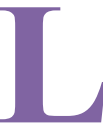

as fracturas de la articulación de la cadera comprenden fracturas del acetábulo, de cabeza femoral y de la porción proximal del fémur. Las fracturas de cadera pueden producirse en individuos de cualquier edad. En pacientes jóvenes, estas fracturas son habitualmente resultado de traumatismos de alta energía, como las colisiones automovilísticas. Estos pacientes a menudo sufren lesiones que afectan a múltiples órganos así como lesiones ortopédicas asociadas. En pacientes ancianos, las lesiones son el resultado de un mecanismo de menor intensidad como una caída; los pacientes mayores representan un colectivo vulnerable en las diferentes esferas que conforman la vida (social, familiar, médica, económica, etc) y a menudo presentan múltiples comorbilidades médicas asociadas (National Center for Injury Prevention and Control, 2019).

En España se producen entre 50.000 y 60.000 fracturas de cadera al año (Alvarez-Nebreda, 2008). En Cataluña el año 2015 la población mayor de 65 años representaba un $17,4 \%$ (1,304 millones) y se espera que llegue al $30 \%$ (2,450 millones) en 2050, lo mismo ocurre con el índice de dependencia de los ancianos, el que va en aumento. Se ha visto que el grupo mayor de 80 años tiene un crecimiento más rápido dentro la población, y se prevé que se acelere en los próximos 50 años. Las causas que justifican este crecimiento es el incremento de la esperanza de vida, la disminución de la mortalidad y el descenso de la tasa de natalidad (IDESCAT, 2016). Las estimaciones indican que la incidencia de fracturas de cadera a nivel mundial alcanzará nada menos que los 6,5 millones por año en 2050 (Riggs, 1995). Una mujer caucásica de 50 años tiene actualmente un riesgo del $17,5 \%$ de sufrir una fractura de cadera a lo largo de su vida; para los varones de la misma edad este porcentaje es del 6\%. En Estados Unidos, la atención a pacientes con fractura de cadera supone una factura anual de más de 14.000 millones de dólares, cantidad que podría alcanzar los 250.000 millones en 2040. En España, los costes directos de tratar una fractura de cadera varían entre los 3000 y los $5200 €$ cada una dependiendo del hospital y de la Comunidad Autónoma. El coste total para las arcas públicas es de entre 300-860 millones de Euros al año, sin tener en cuenta los costes adicionales indirectos del cuidado de los pacientes, los gastos de convalecencia en residencias de ancianos y la rehabilitación posterior (Alvarez-Nebreda, 2008). 
Los problemas de la marcha y del equilibrio son comunes en las personas mayores y contribuyen al riesgo de caída y de lesiones. Estudios prospectivos indican que el 30\% de la población mayor de 65 años sufre alguna caída al año, la proporción es mayor en pacientes frágiles e ingresados en residencias y centros sociosanitarios (Moran, 2005).

En este colectivo es muy frecuente la osteoporosis, un proceso crónico que aparece con la edad y que está influenciado por factores hereditarios, ambientales y de estilo de vida. Es más prevalente en mujeres postmenopáusicas, pero también afecta a hombres y mujeres con trastornos subyacentes o factores de riesgo relacionados con la desmineralización ósea. Las principales manifestaciones clínicas son las fracturas vertebrales y las de cadera. Se define como una reducción de la masa ósea y un deterioro de la microestructura esquelética, que dan lugar a una disminución de la resistencia ósea, un incremento de la fragilidad y un mayor riesgo de fractura. El incremento de la morbilidad, del riesgo de sufrir nuevas fracturas y de la mortalidad son las complicaciones clínicas asociadas a la fractura osteoporótica, las cuales representan un gran impacto clínico, social y económico.

La fractura de cadera en el paciente anciano es la causa más frecuente de ingreso hospitalario en los servicios de traumatología y ortopedia. Cataluña es una de las comunidades autónomas con mayor prevalencia e incidencia de fractura de cadera en ambos sexos. Entre el año 2000 y el 2002 del total de pacientes con fracturas de cadera que tuvieron lugar en España (119.021), el 19,5\% (23.187) se produjeron en Cataluña, y de éstas, el 91,6\% de (21.250) eran en pacientes mayores o igual a 65 años. La incidencia anual crece exponencialmente con la edad, y la incidencia en las mujeres casi triplica la de los homes (Alvarez-Nebreda, 2008). En la actualidad se han considerado como factores etiológicos favorecedores: los cambios nutricionales y metabólicos (déficit de vitamina D), la comorbilidad secundaria a otras enfermedades, una insuficiente actividad física (atrofia muscular), el consumo de tóxicos y la polimedicación. La edad es un factor importante ya que el $85,4 \%$ del total de fracturas de fémur se producen en mayores de 75 años. Éstos son enfermos en los que vemos una repercusión funcional en la deambulación y en las actividades de la vida diaria, y una repercusión cognitiva no sólo porque la mayoría sufren alguna demencia, sino también porque en la mitad de los casos aparece síndrome confusional 
(en ocasiones denomidado delirium) durante la hospitalización. También aumenta significativamente la probabilidad de muerte, que es mayor durante los meses posteriores a la fractura y que al año es, aproximadamente, del 20-30\%. Según las estimaciones realizadas por diversos autores, sufrir una fractura de cadera acorta entre 2 y 4 años la expectativa de vida (Vidan, 2013).

Para paliar este problema y mejorar la atención del paciente geriátrico, en los años 60, en Inglaterra, se iniciaron los primeros sistemas de colaboración entre el servicio de traumatología y el de geriatría en los pacientes que habían padecido fractura de cadera (Vidan, 2013; Brauer, 2009). Consistían en Unidades de Ortogeriatría (UOG) para pacientes en fase subaguda, es decir, aquella fase en la que paciente ya había sido tratado quirúrgicamente y precisaba de un periodo de rehabilitación y convaslencia postoperatorio. Posteriormente se extendió en la fase aguda del enfermo desde el primer momento de ingreso y previo a cualquier tratamiento quirúrgico. Este nuevo modelo consiste en un equipo multidisciplinar de atención al enfermo en su fase aguda, que está formado por traumatología, geriatría, anestesista, rehabilitador/a, fisioterapeutas y terapeutas ocupacionales, trabajador / a social, enfermería y auxiliares de enfermería. El objetivo es iniciar de forma precoz la atención al enfermo, mejorar la evolución clínica reduciendo la mortalidad, rehabilitación precoz, mejorar el pronóstico funcional, reducir la estancia hospitalaria y reducir los gastos de la asistencia.

En algunos estudios se ha visto que los pacientes tratados en UOG realizan más frecuentemente rehabilitación intrahospitalaria durante la fase aguda, su situación funcional al alta es mejor y con más frecuencia son derivados a las unidades de recuperación funcional. También se reduce la estancia en hospital de agudos y la estancia hospitalaria total (agudos y media estancia). Esto por lo tanto también implica un menor gasto hospitalario, mayor efectividad y eficiencia (González, 2011; Baztán, 2011).

\section{Objetivos del Estudio}

El objetivo principal de este análisis es evaluar la dependencia para las Actividades Básicas de la Vida diaria como factor pronóstico después de 
sufrir una fractura de cadera y evaluar algunas de las complicaciones que aparecen durante el ingreso hospitalario (delirium activo, impactación fecal, incontinencia de orina, llagas, infección respiratoria, disfagia, broncoaspiración y necesidad de transfusión de sangre), así cómo, algunas de las complicaciones post-quirúrgicas más frecuentes (anemia, estreñimiento, ITU, infección de la herida quirúrgica) para ver como influyen en la evolución posterior de los pacientes.

\section{Metodología}

En los siguientes apartados se describe la metodología utilizada para la realización de este análisis.

\section{Diseño, Población y Muestra}

Es un estudio de casos retrospectivo realizado a partir de la revisión de las historias clínicas de 428 pacientes. El periodo de estudio está situado entre el 29 de Diciembre de 2015 y el 27 de Julio de 2017 ambos incluidos. La población estudiada incluyó a todos los pacientes $\geq 65$ años que ingresaron en la Unidad de Ortogeriatría del Hospital Universitario Sant Joan de Reus con diagnóstico de fractura de cadera. La lista de todos estos pacientes se ha obtenido a través de la secretaría del Área de Atención Sociosanitaria y Geriatría del hospital.

\section{Criterios Exclusión e Inclusión}

Se han excluido del estudio todos aquellos pacientes que:

1. No eran $\geq 65$ años.

2. Su tipo de fractura no era osteoporótica. Se ha considerado fractura osteoporótica aquella producida por un traumatismo de baja energía.

3. No presentaban una fractura de cadera. Se ha considerado fracturas de cadera todas aquellas que eran de fémur proximal según la clasificación de la $\mathrm{AO}$ foundation.

4. Estaban repetidos en la lista de pacientes en estudio.

5. Firmaron el consentimiento de traslado a su país para ser operados. 
6. No siguieron seguimiento mínimo de 12 meses por parte de la Unidad de Ortogeriatría.

7. No pertenecían a la región sanitaria propia de nuestro centro.

8. Aquellos donde el registro de todos los datos para realizar el estudio no se hubiese completado correctamente o dentro de los plazos establecidos.

\section{Variables Principales y Secundarias}

Como variables principales se ha considerado la fractura osteoporótica de cadera y ser mayor o igual de 65 años. Como variables secundarias se han considerado: edad, sexo, IMC, lugar de procedencia, polimedicación, tipo de fractura osteoporótica, diagnóstico previo de osteoporosis, tratamiento osteoporótico previo, tratamiento previo con vitamina $\mathrm{D}$, vitamina $\mathrm{D}$, calcio sérico, TSH, PTH, creatinina, hemoglobina, fracturas osteoporóticas previas, deterioro cognitivo, tabaquismo, alcoholismo, delírium activo, impactación fecal, incontinencia orina, llagas, infección respiratoria, disfagia, broncoaspiración, transfusión de sangre, anemia, infección de la herida quirúrgica, ITU (infección del tracto urinario), estreñimiento, destino al alta, índice de Barthel, índice de Lawton y Brody y índice de Pfeiffer.

Para valorar el grado de dependencia se han recogido los valores de dos escalas. La primera, es la escala de Lawton y Brody que nos evalúa las actividades instrumentales de la vida diaria. La segunda es el índice de Barthel, que nos evalúa las actividades básicas de la vida diaria, de la que se recogieron las puntuaciones anotadas antes del ingreso, al ingreso y al alta. Finalmente, para valorar el deterioro cognitivo se han recogido los valores obtenidos a partir del cuestionario de Pfeiffer y también se ha tenido en cuenta si previamente fueron diagnosticados de alguna demencia, si no fueron diagnosticados de demencia o algún otro trastorno neurodegenerativo pero tenían un deterioro cognitivo y si durante la estancia hospitalaria desarrollaron un deterioro cognitivo. Toda esta información se ha obtenido del informe de alta de la Unidad de Geriatría y de los apuntes realizados por todos los profesionales sanitarios registrados en la historia clínica del enfermo.

Para el análisis, se ha destacado únicamente el Índice de Barthel para valorar el grado de funcionalidad y autonomía del paciente y relacionarlo 
con la mortalidad y otros factores de riesgo. Se ha tomado esta decisión por los siguientes motivos: es el test más utilizado por otros autores en la bibliografía disponible en bases de datos y se trata de un test muy extendido en práctica habitual del manejo geriátrico, lo que permite poder comprar resultados con la gran mayoría de centros alrededor del mundo. El test de Barthel desarollado por Collin en 1988 (también conocido como Índice de Discapacidad de Mayland) es una escala que mide la capacidad de una persona para realizar 10 actividades habituales en la vida diaria y que se consideran básicas, midiendo así su grado de independencia. Se interroga al paciente sobre cada una de las actividades correspondientes (comer, aseo personal, uso del retrete, desplazarse, subir y bajar escaleras, vestirse y desvestirse, trasladarse entre la silla y la cama, bañarse o ducharse, control de heces y control de orina) y según su capacidad para llevarla a cabo se le otorga una puntuación de 0,5 y 10 puntos (en algunas actividades hasta 15 puntos), con una puntuación máxima de 100 puntos. En el caso que el paciente utilice habitualmente sillas de ruedas la puntuación máxima será de 90 puntos. Se considera que el paciente que adquiere 100 puntos en el test es un paciente autónomo e independiente, entre 99-91 puntos presenta una dependencia leve, entre 90-61 puntos una dependencia moderada, entre 60-21 una dependencia grave y por debajo de 20 puntos, una dependencia total para las actividades básicas de la vida diaria (Rozman, 2016).

Del curso clínico de servicio de Cirugía Ortopedica y Traumatología se recogió el tipo de fractura osteoporótica y se clasificó según la codificación de la Clasificación de Müller de la AO Foundation. Esta clasificación permite codificar el tipo de fractura con un código alfanumérico y rompe las barreras idiomáticas entre traumatológos de todo el mundo. Dentro del grupo de las fracturas de cadera extraarticulares incluye las fracturas de macizo trocanterico, las pertrocantèricas y las persubtrocantèricas; y dentro del grupo de las fracturas intraarticulares incluye las de cuello de fémur, las subcapitales y las transcervicales.

Las variables englobadas dentro de las complicaciones durante el ingreso (delirium activo, impactación fecal, incontinencia orina, llagas, infección respiratoria, disfagia, broncoaspiración y transfusión de sangre) y dentro de las complicaciones post-quirúrgicas (infección herida, ITU y estreñimiento) se han obtenido del informe de alta y de la revisión de todos los cursos clínicos escritos por el personal sanitario responsable del 
enfermo. La variable anemia se ha valorado de acuerdo con los valores analíticos obtenidos de la $\mathrm{Hb}$ y utilizando como valores de referencia los del libro Harrison-Principios de Medicina Interna (Longo, 2012).

\section{Métodos Estadísticos}

Las variables continuas se muestran en media y desviación estándar, y las categóricas en porcentaje.

Para el análisis de variables categóricas se ha empleado la prueba Xi cuadrado de Pearson y los factores asociados a mortalidad mediante regresiones logísticas.

Se ha establecido un punto de significación estadística de $\mathrm{P}<0,05$.

El análisis de datos se ha realizado con el programa estadístico IBM SPSS Statistics 23.

\section{Resultados}

Se ha visto una diferencia importante en el sexo, el $73,8 \%$ de la población son mujeres, mientras que sólo el $26,2 \%$ son hombres, por lo tanto la población más representativa es el sexo femenino. La edad media de la población es de 84,62 \pm 7,7 años, sin apreciarse una gran diferencia entre mujeres y hombres, la cual es de $85,83 \pm 7,9$ años y $83,54 \pm 6,7$ años respectivamente. De los pacientes ingresados por fractura de fémur proximal, un $56 \%$ sufrió fractura intracapsular, mientras que un $44 \%$ sufrió fractura extracapsular, sin encontrar diferencias significativas por sexo o grupo de edad.

Un 43,6\% de los pacientes presentaban dependencia grave el ingreso según la escala de Barthel. La media de puntuación de la escala de Barthel previa al ingreso fue de 60,78 puntos $(\mathrm{s}=23,25)$ y al alta de 37,39 puntos ( $\mathrm{s}$ $=22,50)$ siendo la diferencia significativa $(\mathrm{p}<0,001)$. El $70 \%$ de los pacientes requirió ingreso en Centro Sociosanitario al alta para realizar convalecencia.

Las complicaciones postoperatorias más comunes registradas fueron la anemia postoperatoria y el síndrome confusional agudo. Cabe destacar en primer lugar que el $83,6 \%$ de la población sufrió anemia después de la cirugía y que la media de la $\mathrm{Hb}$ post-quirúrgica fue de 9,92 g / dl. Cómo se 
recogieron los valores analíticos de la $\mathrm{Hb}$ al ingreso y de la $\mathrm{Hb}$ postquirúrgica, se pudo comparar ambas y se pudo observar que existe una diferencia significativa entre $\mathrm{la} \mathrm{Hb}$ al ingreso y la $\mathrm{Hb}$ post-quirúrgica.El estreñimiento se presentó en un $25 \%$ de la población en estudio, la infección del tracto urinario en un $12,1 \%$ y la infección de la herida quirúrgica sólo en un $2,6 \%$.

El 55,2\% de los pacientes analizados tomaban de forma habitual más de 5 fármacos diferentes diariamente. La mortalidad a los 6 meses de seguimiento fue del $24 \%$ de los pacientes y el año del $33,5 \%$. El estudio de regresión logística muestra que sufrir dependencia grave en la escala de Barthel al ingreso es factor de riesgo de muerte a los 6 meses $(p=0,047)$ y el año $(\mathrm{p}=0,043)$. La dependencia grave al alta también se asocia a la mortalidad a los 6 meses $(\mathrm{p}<0,001)$. Padecer síndrome confusional agudo durante el ingreso es un factor de riesgo de muerte al año $(p=0,041)$. Por otra parte, no sufrir dependencia es un factor protector de mortalidad a los 6 meses $(p=0,023)$.

\section{Discusión}

El estado funcional, la calidad de vida, la institucionalización y la mortalidad son variables de resultado fundamentales a la hora de evaluar la asistencia que se ofrece al paciente geriátrico (Torres Moreno, 2009). La funcionalidad y autonomía son considerados indicadores de salud que condicionan el pronóstico y la evolución posterior del paciente anciano frágil, más importante, en algunas ocasiones, que el diagnóstico principal por el cual son ingresados. La secuencia que conduce a la discapacidad y la dependencia en personas de edad se relaciona con múltiples causas. Algunas agudas como la enfermedad cerebrovascular aguda y la fractura de cadera, principalmente; y otras crónicas e insidiosas como la enfermedad de Alzheimer, la demencia vascular, enfermedades articulares y del aparato locomotor, cardiopatías, diabetes, deterioro sensorial o síndromes neurológicos, entre otros, que se ven influenciadas a su vez por factores del entorno en el que se desarrolla la vida cotidiana de cada anciano.

Es importante remarcar, por otro lado, que la comorbilidad es habitual en los pacientes geriátricos y tiene a aumentar con la edad. Hasta el $24 \%$ de los mayores de 65 años y hasta el $31 \%$ de los mayores de 85 años presentan 
4 o más diagnósticos de enfermedades de larga evolución (Bartke, 2001). Es por esta razón, que habitualmente, el paciente anciano recibe tratamientos con múltiples fármacos para tratar cada una de las patologías que aparecen con el envejecimiento. La polimedicación, definida como la toma de más de 5 fármacos diferentes al día por un paciente de forma continuada durante más de un año, esta relacionada con un aumento del número de caídas y un incremento de ingresos hospitalarios. En la muestra estudiada, el 55,2\% de los pacientes presentaban condición de paciente polimedicado en el momento del ingreso. Shih-Wei Lai, en su análisis poblacional de 2328 individuos sobre polifarmacia y fracturas de cadera, concluye que el riesgo de fractura de cadera aumenta con el número de fármacos utilizados, especialmente en población femenina (Lai, 2010).

La mortalidad a un año para las fracturas de cadera oscila entre el 14\% y el $36 \%$, unas cifras significativas considerando la prevalencia de tales lesiones. En nuestro análisis, la mortalidad a los 6 meses de seguimiento fue del $24 \%$ de los pacientes y el año del $33,5 \%$, similar a otros estudios revisados a la bibliografia7. En los estudios de mortalidad asociada a fractura de cadera se han sugerido varios factores asociados al aumento de la mortalidad en pacientes ancianos tras fractura de cadera, principalmente la edad, el género masculino, las comorbilidades, la demora de la intervención, el nivel de autonomía previo y el estado cognitivo. La edad es el factor de riesgo de mortalidad más frecuentemente descrito en la mayoría de estudios sobre población española. Sin embargo, esos estudios están basados en series de casos de pacientes afectados de fractura de cadera con rangos de edad bastante amplios, que van desde los 60 a los 102 años. Además, la mayoría no estratificaban los pacientes de acuerdo con el tipo de fractura de cadera u otras variables (Novoa-Parra, 2019).

En la población española se han reportado diferentes factores de riesgo de mortalidad en diferentes estudios. En cuanto a nuestro análisis hemos encontrado un vínculo importante entre la dependencia y la mortalidad. En el trabajo llevado a cabo Novoa-Parra (2019), se considera el índice de Barthel como el factor más sensible de predictor de mortalidad al año en población mayor de 80 años. Este autor, reporta un aumento de riesgo de muerte al año en aquellos pacientes mayores de 80 años que presentan una puntuación inferior a 85 puntos en el indice de Barthel registrada al ingreso del paciente. En la serie estudiada por Torres-Moreno (2009), se destacó el 
índice de Barthel y el índice de Charlson como factores predictores de mortalidad. La correlación con la mortalidad parece fuerte, no así como factores predictores de recuperación funcional posterior (Burgos, 2008; Perez Bocanegra, 2016).

Aranguren et al. (2017) identificaron el índice de Barthel como predictor de mortalidad a 90 días, 1 y 2 años, estableciendo un punto de corte de 60 puntos por lo que separaban los pacientes con dependencia severa y total de aquellos con menores grados de dependencia. En el estudio de Folbert et al. (2016) sobre fracturas de cadera en general, además del estado funcional valorado con el índice de Barthel, encontraron como variables asociadas a la mortalidad al año de seguimiento el género masculino , la edad avanzada, una puntuación ASA de III o mayor, puntuación alta en el índice de Charlson, la malnutrición y las limitaciones físicas previas. Por el contrario, Blay et al. (2018) observaron que el índice de Barthel no era significativo de mortalidad al año tras fractura de cadera. El estudio de regresión logística llevado a cabo en nuestro análisis muestra que sufrir dependencia grave en la escala de Barthel al ingreso es factor de riesgo de muerte a los 6 meses $(p=0,047)$ y el año $(p=0,043)$ y que la dependencia grave al alta también se asocia a la mortalidad a los 6 meses $(\mathrm{p}<0,001)$, obteniendo resultados similares a los descritos por otros autores en la bibliografía revisada.

Es importante destacar cómo la presencia de síndrome confusional agudo o delirio durante el ingreso condiciona el estado clínico global de los pacientes y su posible asociación independiente con los resultados funcionales. El delirio es un problema psiquiátrico frecuente entre los ancianos que, en numerosas ocasiones, es pasado por alto por los profesionales de la salud. Entre el 10\%-15\% de ancianos ingresados en un hospital por una enfermedad médica aguda presenta un cuadro confusional agudo. Un 5\%-30\% adicional lo desarrolla durante su estancia hospitalaria a menudo como consecuencia de la yatrogenia. Entre los ancianos frágiles la prevalencia de esta entidad puede superar el 50\%. El delirio puede tener un impacto negativo en el estado general de la salud, de tal manera que multiplica por dos la mortalidad de los pacientes que desarrollan esta entidad. En nuestro análisis, sufrir síndrome confusional agudo durante el ingreso es un factor de riesgo de muerte al año $(p=0,041)$. Guerini et al (2010), mostraron en sus investigaciones, cómo la presencia de síndrome 
confusional agudo en el momento de la admisión a un entorno hospitalario se asocia significativamente con peores resultados funcionales a la alta evaluados con el Index total de Barthel. El delirio es un síndrome transitorio de comienzo agudo, potencialmente reversible, que manifiesta una disfunción cerebral de la que se desconoce su etiología pero de la que se han identificado diferentes grupos causales: alteraciones intracraneales primarias, enfermedades sistémicas, agentes tóxicos exógenos y factores ambientales. Es necesario un alto indice de sospecha, sobre todo en los pacientes de alto riesgo, ya que condiciona su pronóstico posterior.

Cabe mencionar que todas guías nacionales e internacionales analizadas (Avellana, 2007; SIGN, 2009; NZCG, 2003; BOA-BGS, 2007) recomiendan la colaboración entre el servicio de geriatría y traumatología para el manejo del paciente anciano en todas las fases de proceso evolutivo de la fractura de cadera y lo hacen con un grado de recomendación A. En las últimas décadas la colaboración entre geriatras y traumatólogos en el manejo de la fractura de cadera ha sido creciente. Esta colaboración comenzó gracias a Michael Devas, un cirujano ortopédico, y Bobby Irvine, un geriatra, en 1960, en Inglaterra. Ambos compartieron la idea de la repercusión que supone para un anciano el padecer una fractura de cadera, la importancia de la recuperación de la función como objetivo principal del tratamiento y la rentabilidad del trabajo conjunto en la búsqueda de dicho objetivo, surgiendo así el concepto de ortogeriatría como forma de mejorar los resultados (Vidán, 2013) Desde el origen de la colaboración geriatríatraumatología en el tratamiento de la fractura de cadera se han desarrollado varios modelos de intervención geriátrica durante las distintas fases de su tratamiento. Aunque diversos estudios concluyen que esta colaboración aporta beneficios sobre el tipo tradicional de tratamiento, no está claro cual de los modelos conlleva mejores resultados (Etxebarria-Foronda, 2015). La intensidad de la intervención en cada uno de ellos ha sido variable $\mathrm{y}$ adaptada a las circunstancias locales y a las características de cada hospital, lo cual dificulta su comparación. En general se han obtenido mayores beneficios cuando la intervención del geriatra comienza desde el momento del ingreso y se realiza de forma intensiva, es decir con visita médica diaria y participación en la toma de decisiones junto con el equipo de traumatología. 
Los principales estudios aleatorizados publicados presentan 4 modelos principales de colaboración:

Paciente ingresado en la planta de traumatología con el geriatra como consultor. Sólo un trabajo, y con escaso número de pacientes, demostró beneficio en cuanto a reducción de la estancia hospitalaria (Kennie, 1988). No se encontraron tampoco beneficios en cuanto a la recuperación funcional.

Paciente ingresado en la planta de Traumatología con visita diaria por parte del geriatra. Este es el modelo más frecuentemente utilizado y con mayor número de estudios que analizan sus resultados (Naglie, 2002; Swanson, 1998; Zuckerman, 1992; Antonelli-Incalzi, 1993; Khan, 2002; Koval, 2004; Fisher, 2006). Globalmente este modelo aporta una reducción ligera de la estancia media aunque este resultado no es una constante en todos los estudios. En dos estudios la intervención del geriatra se asocia a menos complicaciones médicas durante el ingreso y en uno de ellos hubo además menor mortalidad en el grupo atendido diariamente por un geriatra.

El paciente con fractura de cadera es ingresado en una planta de Geriatría y con el traumatólogo actuando como consultor. Es el modelo de colaboración más infrecuente de todos. El resultado conjunto de estos estudios demostró una reducción en la estancia hospitalaria y un estudio (Stenvall, 2007) encontró beneficios en la capacidad funcional al año de la fractura.

Paciente ingresado en la planta de Traumatología y con cuidados integrados por parte de un equipo de geriatría (Friedman, 2009; Shyu, 2008; Khasraghi, 2005; González-Montalvo, 2010). Es el modelo que ha demostrado mayores beneficios. El equipo de geriatría, compuesto por médico, enfermera y trabajador social, trabaja conjuntamente con el equipo de traumatología durante todo el ingreso, compartiendo responsabilidad y decisiones. Este modelo tiene los resultados medios mejores en cuanto a reducción de la mortalidad hospitalaria, menor tiempo hasta la cirugía, menor estancia media global y menor tasa de complicaciones postoperatorias.

En nuestro centro, nos organizamos como unidad de Ortogeriatría, donde se incluye geriatra, reumatólogo, anestesista, equipo de rehabilitación y traumatólogo en un único equipo para coordinar todo el proceso. Además del respaldo científico que sostiene este tipo de 


\section{García et al. - Factor Pronóstico de Fractura de Cadera}

intervención, consideramos que es la mejor manera de evaluar y gestionar posteriormente todos los recursos médicos, sociales y familiares que comporten una mejor evolución del paciente, una mejor atención a la dependencia y, por tanto, un mejor pronóstico posterior. Como resultado provisional, desde la aplicación de la UOG en nuestro centro se ha conseguido una disminución de la mortalidad intrahospitalaria del 3\% al $1 \%$ y el porcentaje de pacientes bajo tratamiento farmacológico para la osteoporosis tras el ingreso ha aumentado del 13\% al 71\%. Consideramos que este aumento en el tratamiento de la osteoporosis puede tener un efecto preventivo de la fractura de cadera y otras fracturas osteoporóticas, disminuyendo la incidencia de éstas y los riesgos de mortalidad asociados a sufrir las mismas.

\section{Conclusión}

En la muestra analizada, sufrir dependencia en el ingreso según el índice de Barthel es un factor de riesgo de muerte a los 6 meses y al año. Por otro lado, no sufrir dependencia según el índice de Barthel es un factor protector de la mortalidad a los 6 meses. Registrar el grado de autonomía del paciente, por lo tanto, aporta una información muy valiosa para el pronóstico del mismo. La organización de Unidades Ortogeriátricas multidisplicinares debe ser el instrumento para paliar el riesgo de mortalidad asociado a la dependencia y la vulnerabilidad.

Sería interesante para futuras investigaciones analizar el impacto que produce la perdida de autonomía sobre los cuidadores principales y familiares de los pacientes afectados por una fractura de cadera y adecuar los recursos necesarios para hacer frente a esta situación.

Tendiendo en cuenta la tendencia del envejecimiento de la población y el aumento de incidencia de esta patología en la sociedad, consideramos la necesidad de desarrollar de forma urgente políticas de prevención de la osteoporosis en población joven (dieta equilibrada, práctica deportiva, evitar el consumo de tóxicos, exposición solar adecuada, etc) y acciones contra la dependencia del colectivo anciano. 


\section{Referencias}

Alvarez-Nebreda, M.L., Jimenéz, A.B., Rodríguez P., \& Serra, J. A. (2008). Epidemiology of hip fracture in the elderly in Spain. Bone, 42(2), 278-285.

Antonelli Incalzi, R., Gemma, A., Capparella, O., Bernabei, R., Sanguinetti, C., \& Carbonin, P.U. (1993). Continuous geriatric care in orthopedic wards: a valuable alternative to orthogeriatric units. Aging; 5, 207-16. Aranguren-Ruiz, M.I., Acha-Arrieta, M.V., Casas-Fernández de Tejerina, J.M., Arteaga-Mazuelas, M., Jarne-Betrán, V., \& Arnáez-Solis, R. (2017). Factores de riesgo de mortalidad tras intervención quirúrgica de fractura de cadera osteoporótica en pacientes mayores. Revista Española de Cirurgía Ortopédica y Traumatología, 61, 185-192. doi: 10.1016/j.recot.2017.02.004

Avellana Zaragoza, J.A. \& Fernández Portal, L. (2007). Guía de buena práctica clínica en Geriatría. Anciano afecto de fractura de cadera. Madrid: SECOT.

Bartke, A., Coschigano, K., Kopchick, J., Chandrashekar, V., Mattison, J., \& Kinney, B. (2001). Genes that prolong life: Relationships of growth hormone and growth to aging and life span. Journal of Gerontology: Biological Sciences, 56(8), 340-349. doi: 10.1093/gerona/56.8.b340

Baztán, J.J., Suárez-García, F.M., López-Arrieta, J., \& Rodríguez-Mañas, L. (2011). Eficiencia de las unidades geriátricas de agudos: metaanálisis de estudios controlados. Revista Española de Cirurgía Ortopédica y Traumatología, 46(4), 186-192.

Blay-Domínguez, E., Lajara-Marco, F., Bernáldez-Silvetti, P.F., VeracruzGálvez, E.M., Muela-Pérez, B., Palazón-Banegas, M.A. et al. (2018). Índice O-POSSUM como predictor de morbimortalidad en pacientes intervenidos de fractura de cadera. Revista Española de Cirugía Ortopédica y Traumatología, 62(3), 207-215. doi: 10.1016/j.recot.2017.10.013

Brauer, C.A., Coca-Perraillon, M., Cutler D.M., \& Rosen, A.B. (2009) Incidence and mortality of hip fractures in the United States. JAMA, 302, 1573-1579. 
British Orthopaedic Association-British Geriatric Society (BOA-BGS) (2007). The care of patients with fragility fracture. Disponible en: https://www.bgs.org.uk/sites/default/files/content/attachment/201805-02/Blue $\% 20$ Book $\% 20$ on $\% 20$ fragility $\% 20$ fracture $\% 20$ care.pdf

Burgos, E., Gómez-Arnau, J.I., Diea, R., Muñoz, L., Fernández-Guisasola,

J., \& García del Valle, S. (2008). Predictive value of six risk scores for outcome after surgical repair of hip fracture in elderly patients. Acta Anaesthesiologica Scandinavica, 52(1), 125-31. doi: 10.1111/j.1399-6576.2007.01473.x

Etxebarria-Foronda, J.R., Caeiro-Rey, R., Larrainzar-Garijo, E., VaqueroCervino, L., Roca-Ruiz, M., Mesa-Ramos, et al. (2015). Guía SECOT-GEIOS en osteoporosis y fractura por fragilidad. Actualización. Revista Española de Cirurgía Ortopédica y Traumatología, 59(6), 373-393. doi: 10.1016/j.recot.2015.05.007

Fisher A.A., Davis, M.W., Rubenach, S.E., Sivakumaran, S., Smith P.N., \& Budge, M.M. (2006). Outcomes for older patients with hip fractures: the impact of orthopedic and geriatric medicine cocare. Journal of Orthoppaedic Trauma, 20(3), 172-180. doi: 10.1097/01.bot.0000202220.88855.16

Folbert, E.C., Hegeman, J.H., Vermeer, M., Regtuijt, E.M., van der Velde, ten Duis, J.J, Slaets, J.P. (2016). Improved 1-year mortality in elderly patients with a hip fracture following integrated orthogeriatric treatment. Osteoporosis International, 28(1), 269-277. doi: 10.1007/s00198-016-3711-7

Friedman, S.M., Mendelson D.A., Bingham, K.W., Kates, S.L. (2009). Impact of a comanaged Geriatric Fracture Center on short-term hip fracture outcomes. Archives of Internal Medicine, 169, 1712-1717. doi: 10.1001/archinternmed.2009.321

González, J.I., Gotor, P., Martín, A. et al. (2011). La unidad de ortogeriatría de agudos. Evaluación de su efecto en el curso clínico de los pacientes con fractura de cadera y estimación de su impacto económico. Revista Española de Geriatría y Gerontología, 46(4), 193-199. doi: 10.1016/j.regg.2011.02.004

González-Montalvo, J.I., Alarcón, T., Mauleón, J.L., Gil-Garay, E., Gotor, P., \& Martín-Vega, A. (2010). The Orthogeriatric Unit for Acute Patients: A new model of care that improves efficiency in the 
management of patients with hip fracture. Hip International, 20, 22935.

Guerini, F., Frisoni, G.B., Morghen, S., Speciale, S., Bellelli, G., \& Trabucchi M. (2010). Clinical instability as a predictor of negative outcomes among elderly patients admitted to a rehabilitation ward. Journal of the American Medical Directors Association, 11, 443-448. doi: 10.1016/j.jamda.2009.10.005

Idescat. Dossiers Idescat (2016). Projeccions de la població a Catalunya 2013-2051, 18, gener 2015. Disponible en: http://www.idescat.cat/cat/idescat/publicacions/cataleg/pdfdocs/dossi er18.pdf.

Idescat (2015). Projeccions de població de Catalunya 2015-2030 (base 2002). Disponible en: http:/ www.idescat.net.

Longo, D., Fauci, A., Kasper, D., Hauser, S., \& Jameson, J. (2012). Principios de Medicina interna de Harrison. $18^{\circ}$ edición. Madrid: Editorial Mc Graw Hill Medical.

Kennie, D.C., Reid, J., Richardson, I.R., Kiamari, A.A., \& Kelt, C. (1988). Effectiveness of geriatric rehabilitative care after fractures of the proximal femur in elderly women: a randomised clinical trial. $B M J$ 297, 1083-6.

Khan, R., Fernandez, C., Kashifl, F., Shedden, R.; Diggory, P. (2002). Combined orthogeriatric care in the management of hip fractures: a prospective study. Annals of the Royal College of Surgeons of England, 84, 122-4.

Khasraghi, F.A., Christmas, C., Lee, E.J., Mears, S.C., \& Wenz, J.F. (2005). Effectiveness of a multidisciplinary team approach to hip fracture management. Journal of Surgical Orthopaedic Advances, 14, 27-31.

Koval, K.J., Chen, A.L., Aharonoff, G.B., Egol, K.A., \& Zuckerman, J.D. (2004). Clinical pathway for hip fractures in the elderly: the Hospital for Joint Diseases experience. Clinical Orthopaedics and Related Research, 425, 72-81.

Lai, S.W., Liao, K.F., Liao, C.C., Muo, C.H., Liu, C.S., \& Sung, F.C. (2010). Polypharmacy correlates with increased risk for hip fracture in the cualquier fidelidadelderly: a population-based study. Medicine (Baltimore), 89(5), 295-299. doi: 10.1097/MD.0b013e3181f15efc 
Naglie, G., Tansey, C., Kirkland, J.L., Ogilvie-Harris, D.J., Detsky, A.S., Etchells, E. et al. (2002). Interdisciplinary inpatient care for elderly people with hip fracture: a randomized controlled trial. Canadian Medical Association Journal, 167, 25-32.

Moran, C.G., Wenn, R.T., Sikand, M., Taylor, A, M. (2005). Early mortality after hip fracture: Is delay before surgery important? Journal of Bone and Joint Surgery (Am), 87-A, 483-489.

National Center for Injury Prevention and Control (2019). Injury Prevention \& Control. Disponible en:

\section{http://www.cdc.gov/ncipc/factsheets/falls.htm}

New Zealand Guidelines Group (NZCG) (2003). Best practice evidencebased guideline. Acute management and immediate rehabilitation after hip fracture amongst people aged 65 years and over. Disponible en: http://www.nzgg.org.nz/system/resources

Novoa-Parra, C.D., Hurtado-Cerezo, J., Morales-Rodríguez, J., SanjuanCerveró, R., Rodrigo-Pérez, J.L., \& Lizaur-Utrilla, A. (2019). Factores predictivos de la mortalidad al año en pacientes mayores de 80 años intervenidos de fractura del cuello femoral. Revista Sociedad Española de Cirugía ortopédica y traumatología, 63(3), 165-252.

Pérez Bocanegra, M.C., Villegas, E., Guallar, A., Rosado, R., \& VilardellTarrés, M. (2006). Utilidad de un nuevo índice pronóstico de mortalidad tras el alta hospitalaria en pacientes mayores de 70 años. Medicina Clínica, 127, 492-483. doi: doi.org/10.1157/13093269

Riggs, B.L. \& Melton, L. (1995). The worldwide problem of osteoporosis:

Insights afforded by epidemiology. Bone, 17, 505-511.

Rozman, C. (2016). Medicina Interna. $18^{\circ}$ edición. Barcelona: Elsevier.

Scottish Intercollegiate Guidelines Network (SIGN) (2009). Management of

hip fracture in older people. A national clinical guideline. SIGN, 111. Edinburgh: SIGN.

Shyu, Y.I., Liang, J., Wu, C.C., Su, J.Y., Cheng, H.S., Chou, S.W. et al. (2008). Interdisciplinary intervention for hip fracture in older Taiwanese: benefits last for 1 year. Journals of Gerontology Series A: Biological Sciences and Medical Science, 63, 92-97.

Stenvall, M., Olofsson, B., Nyberg, L., Lundstrom, M., \& Gustafson, Y. (2007). Improved performance in activities of daily living and mobility after a multidisciplinary postoperative rehabilitation in older 
people with femoral neck fracture: a randomized controlled trial with 1-year follow-up. Journal of Rehabilitation Medicine, 39, 232-238.

Swanson, C.E., Day, G.A., Yelland, C.E., Broome, J.R., Massey, L., Richardson, H.R. et al. (1998). The management of elderly patients with fe- moral fractures. A randomised controlled trial of early intervention versus standard care. The Medical Journal of Australia 169, 515-518.

Torres Moreno, B., Núñez González, E., Pérez Hernández, D., Simón Turriate, J., Alastuey Giménez, C., Díaz Melián, J. et al. (2009). Índice de Charlson versus índice de Barthel como predictor de mortalidad e institucionalización en una unidad geriátrica de agudos y media estancia. Revista Española de Geriatría y Gerontología, 44, 209-212. doi: 10.1016/j.regg.2009.02.002

Vidán, M.T. \& Vaquero, J. (2013). Unidades de ortogeriatría: Impacto en los resultados de la fractura de cadera en el anciano. Madrid: Monografías SECOT.

Zuckerman, J.D., Sakales, S.R., Fabian, D.R., \& Frankel, V.H. (1992). Hip fractures in geriatric patients. Results of an interdisciplinary hospital care program. Clinical Orthopaedics and Related Research, 274, 213-225.

Javier García Alba, cirujano ortopédico, Hospital Universitario Sant Joan de Reus (HUSJR)

Ivan Dot Pascuet, cirujano ortopédico, HUSJR Alfredo Rodríguez Gangoso, cirujano ortopédico y jefe del servicio de cirugía ortopédica y traumatología, HUSJR

Gabriel De Febrer Martínez, geriatría y jefe de servicio de servicio de geriatría, HUSJR. Universitat Rovira i Virgili, Departamento de medicina y cirugía.

Avinguda del Doctor Josep Laporte, 2, 43204 Reus, Tarragona. 